\title{
Hydrogen Production from Palmitic Acid through Autothermal Reforming: Thermodynamic Analysis
}

\author{
Tawiwan Kangsadan ${ }^{1}$, Thanarak Srisurat ${ }^{1}$, Pattaraporn $\mathrm{Kim}^{2}$, Navadol Laosiripojana ${ }^{3}$, \\ Sunisa Jindasuwan ${ }^{4}$, and Unalome W. Hartley ${ }^{1, *}$
}

1 The Sirindhorn International Thai-German Graduate School of Engineering, King Mongkut's University of Technology North Bangkok, Bangkok 10800, Thailand

2 Chemical Engineering Department, Faculty of Engineering, Mahidol University, Thailand

3 The Joint Graduate School of Energy and Environment, King Mongkut's University of Technology

Thonburi, Bangkok 10140, Thailand

4 Department of Industrial Chemistry, Faculty of Applied Science, King Mongkut's University of

Technology North Bangkok, Bangkok 10800, Thailand

*E-mail: unalome.w.cpe@tggs-bangkok.org

\begin{abstract}
This work studies thermodynamic analysis of hydrogen production via autothermal reforming of palmitic acid. A Gibbs free energy minimization method was applied to analyze thermodynamic of syngas production via oxidative reforming of palmitic acid. Equilibrium compositions were estimated at temperature of $800^{\circ} \mathrm{C}, 900^{\circ} \mathrm{C}$ and $1000^{\circ} \mathrm{C}$ under atmospheric pressure. Optimal operating temperature and molar feed ratios of steam to palmitic acid $(\mathrm{S} / \mathrm{C})$ and oxygen to palmitic acid $(\mathrm{O} / \mathrm{C})$ ranging from 0.5 to 4 ; were determined. The PTC Mathcad Prime 2.0 is used to calculate enthalpy, entropy and Gibbs free energy. Aspen Plus program was applied for calculation of product yields and heat duty. Maximum $\mathrm{H}_{2}$ yield of 17.88 mole/molepalmitic with $\mathrm{S} / \mathrm{C}$ of 4 and $\mathrm{O} / \mathrm{C}$ of 0.5 can be achieved at $1000^{\circ} \mathrm{C}$ and $\mathrm{CO}$ yield were 7.75 mole/molepalmitic with $\mathrm{S} / \mathrm{C}$ of 4 and $\mathrm{O} / \mathrm{C}$ of 4 . Production of hydrogen and carbon monoxide increased with increasing temperature.
\end{abstract}

Keywords: Autothermal reforming, palmitic acid, hydrogen production.

ENGINEERING JOURNAL Volume 19 Issue 4

Received 30 September 2014

Accepted 30 January 2015

Published 31 July 2015

Online at http://www.engj.org/

DOI:10.4186/ej.2015.19.4.153 


\section{Introduction}

Oil crisis and shortage of fossil fuels have stimulated the exploration of alternative energy sources. Hydrogen is an attractive source of renewable energy [1] due to its high energy density by weight. Hydrogen is a combustible gas, potentially a clean energy source and industrially used as a precursor in the production of chemicals.

Hydrogen can be produced by several methods including gasification (of feedstocks such as coal, natural gas and biomass), reforming (autothermal reforming (ATR) and steam reforming (SR)), electrolysis of water and fermentation processes [2]. SR is a strongly endothermic reaction where hydrocarbons react with steam while ATR is a reaction between hydrocarbons, steam and other oxygenated compound; such as hydrogen peroxide, oxygen or air. During ATR, partial oxidation (PO) occurs when the system is stoichiometrically oxygen deficient. PO is an exothermic partial combustion of the fuel and oxygenated gas. In this process, heat released during the partial oxidation can be utilized in the steam reforming, supplying the endothermic energy requirement. This will potentially provide a thermally efficient approach when the thermoneutral condition is applied for heat integration in the ATR.

The renewable energy is one of the most promising energies for the future since it can be renewed environmentally-friendly. The exploitation of renewable fuels to fulfill our energy requirements such as hydrogen, bioethanol, dimethyl ether, and biodiesel has been widely investigated to sustain the energy requirement. Palmitic acid $\left(\mathrm{C}_{16} \mathrm{H}_{32} \mathrm{O}_{2}\right)$ is a heavy hydrocarbon obtained as a byproduct during biodiesel production. Palmitic acid can be a potential source of hydrogen. However, only a few researchers have presented the catalytic reforming or cracking of palmitic acid to hydrogen [3-7]. In this present study, the autothermal reforming processes of palmitic acid to produce hydrogen. The previous study utilized hydrogen peroxide in autothermal reformer for hydrogen production for application in micro fuel cells. Concentration of carbon monoxide in the product gas was minimized [8]. Palmitic acid steam reforming reaction contains of several minor reactions, for instance, alkanes and alkenes reforming reactions [9]. Steam was fed to reform hydrocarbons and to increase $\mathrm{H}_{2}$ to $\mathrm{CO}$ ratio [10]. Oxygen was added as a coreactant in autothermal or oxidative reforming operation, to reduce carbon formation and required external heat. However, the oxygen decreases the $\mathrm{H}_{2}$ to $\mathrm{CO}$ ratio unavoidably. In this paper, the thermodynamic analysis of hydrogen production via oxidative reforming of palmitic acid using steam and oxygen is simulated. A total Gibbs free energy minimization method was adopted to estimate the equilibrium composition at temperatures of 800,900 and $1000^{\circ} \mathrm{C}$.

\section{Methods}

Either (1) equilibrium constant calculation method or (2) minimization of the total Gibbs free energy method should be implemented to predict thermodynamic behavior of the hydrogen production via palmitic acid autothermal reforming using oxygen. In this study, list of possible reactions; such as, alkanes and alkenes steam reforming, water-gas shift, carbon formation [11], coke gasification and Boudouard reaction (disproportionation of carbon monoxide to carbon dioxide and carbon); are summarized. Minimization of the Gibbs free energy is widely utilized in fuel reforming processes and favorable when the reaction is considered at specific temperature and pressure. To compare with the calculation of equilibrium constant method, the Gibbs free energy minimization method is simpler as the former method requires associated chemical reaction information such as enthalpy, entropy and heat capacity of each component. Therefore, the Gibbs free energy minimization method is applied in this work.

\subsection{Autothermal Reforming of Palmitic Acid and Its Side Reactions}

The autothermal reforming of palmitic acid has numerous undesirable side reactions because the palmitic acid is a high number of carbon atoms fatty acid. The possible reaction routes of palmitic acid autothermal reforming are shown in Table 1. Seventeen species including palmitic acid $\left(\mathrm{C}_{16} \mathrm{H}_{32} \mathrm{O}_{2}\right)$, water $\left(\mathrm{H}_{2} \mathrm{O}\right)$, oxygen $\left(\mathrm{O}_{2}\right)$, hydrogen $\left(\mathrm{H}_{2}\right)$, carbon monoxide $(\mathrm{CO})$, carbon dioxide $\left(\mathrm{CO}_{2}\right)$, methane $\left(\mathrm{CH}_{4}\right)$, alkane ( $\mathrm{C}_{n} \mathrm{H}_{2 n+2}$ ), alkene $\left(\mathrm{C}_{n} \mathrm{H}_{2 n}\right)$ and elemental carbon (graphite, $\mathrm{C}$ ) as a solid were included in the simulation. Not that only gas reaction $\left(\mathrm{C}_{1}\right.$ to $\left.\mathrm{C}_{5}\right)$ is considered for alkane and alkene [9]. 
Table 1. Reaction of autothermal reforming using palmitic acid [2].

\begin{tabular}{|c|c|c|}
\hline No. & Reaction & Equation \\
\hline 1 & Steam reforming of palmitic acid & $\mathrm{C}_{16} \mathrm{H}_{32} \mathrm{O}_{2}+14 \mathrm{H}_{2} \mathrm{O} \rightarrow 16 \mathrm{CO}+30 \mathrm{H}_{2}$ \\
\hline 2 & Partial oxidation of palmitic acid & $\mathrm{C}_{16} \mathrm{H}_{32} \mathrm{O}_{2}+15 \mathrm{O}_{2} \rightarrow 16 \mathrm{CO}+16 \mathrm{H}_{2} \mathrm{O}$ \\
\hline 3 & Overall reaction of palmitic acid & $\mathrm{C}_{16} \mathrm{H}_{32} \mathrm{O}_{2}+7 \mathrm{H}_{2} \mathrm{O}+7.5 \mathrm{O}_{2} \rightarrow 16 \mathrm{CO}+15 \mathrm{H}_{2}+8 \mathrm{H}_{2} \mathrm{O}$ \\
\hline 4 & Methane decomposition & $\mathrm{CH}_{4} \rightarrow \mathrm{C}+2 \mathrm{H}_{2}$ \\
\hline 5 & Boudouard reaction & $2 \mathrm{CO} \rightarrow \mathrm{C}+\mathrm{CO}_{2}$ \\
\hline 6 & Water-gas shift reaction & $\mathrm{CO}+\mathrm{H}_{2} \mathrm{O} \rightarrow \mathrm{CO}_{2}+\mathrm{H}_{2}$ \\
\hline 7 & $\begin{array}{l}\text { Hydrogenation of carbon } \\
\text { monoxide(1) }\end{array}$ & $\mathrm{CO}+3 \mathrm{H}_{2} \rightarrow \mathrm{CH}_{4}+\mathrm{H}_{2} \mathrm{O}$ \\
\hline 8 & Hydrogenation of carbon dioxide & $\mathrm{CO}_{2}+4 \mathrm{H}_{2} \rightarrow \mathrm{CH}_{4}+2 \mathrm{H}_{2} \mathrm{O}$ \\
\hline 9 & $\begin{array}{l}\text { Hydrogenation of carbon } \\
\text { monoxide }(2)\end{array}$ & $2 \mathrm{CO}+2 \mathrm{H}_{2} \rightarrow \mathrm{CO}_{2}+\mathrm{CH}_{4}$ \\
\hline 10 & Hydrogenation of coke & $\mathrm{C}+2 \mathrm{H}_{2} \rightarrow \mathrm{CH}_{4}$ \\
\hline 11 & Methane steam reforming & $\mathrm{CH}_{4}+\mathrm{H}_{2} \mathrm{O} \rightarrow \mathrm{CO}+3 \mathrm{H}_{2}$ \\
\hline 12 & Methane dry reforming & $\mathrm{CH}_{4}+\mathrm{CO}_{2} \rightarrow 2 \mathrm{CO}+2 \mathrm{H}_{2}$ \\
\hline 13 & Carbon monoxide reduction(1) & $\mathrm{CO}+\mathrm{H}_{2} \rightarrow \mathrm{C}+3 \mathrm{H}_{2} \mathrm{O}$ \\
\hline 14 & Carbon monoxide reduction(2) & $2 \mathrm{CO}+2 \mathrm{H}_{2} \rightarrow 2 \mathrm{C}+2 \mathrm{H}_{2} \mathrm{O}$ \\
\hline 15 & Reverse water-gas shift reaction & $\mathrm{CO}_{2}+\mathrm{H}_{2} \rightarrow \mathrm{CO}+\mathrm{H}_{2} \mathrm{O}$ \\
\hline 16 & Coke gasification & $\mathrm{C}+\mathrm{H}_{2} \mathrm{O} \rightarrow \mathrm{CO}+\mathrm{H}_{2}$ \\
\hline 17 & Oxidation of methane & $\mathrm{CH}_{4}+2 \mathrm{O}_{2} \rightarrow \mathrm{CO}_{2}+2 \mathrm{H}_{2} \mathrm{O}$ \\
\hline 18 & Partial oxidation of carbon monoxide & $\mathrm{CO}+0.5 \mathrm{O}_{2} \rightarrow \mathrm{CO}_{2}$ \\
\hline
\end{tabular}

\begin{tabular}{|c|c|c|}
\hline \multicolumn{3}{|c|}{ Alkane steam reforming } \\
\hline 19 & Methane SR & $\mathrm{CH}_{4}+\mathrm{H}_{2} \mathrm{O} \rightarrow \mathrm{CO}+3 \mathrm{H}_{2}$ \\
\hline 20 & Ethane SR & $\mathrm{C}_{2} \mathrm{H}_{6}+2 \mathrm{H}_{2} \mathrm{O} \rightarrow 2 \mathrm{CO}+5 \mathrm{H}_{2}$ \\
\hline 21 & Propane SR & $\mathrm{C}_{3} \mathrm{H}_{8}+3 \mathrm{H}_{2} \mathrm{O} \rightarrow 3 \mathrm{CO}+7 \mathrm{H}_{2}$ \\
\hline 22 & Butane SR & $\mathrm{C}_{4} \mathrm{H}_{10}+4 \mathrm{H}_{2} \mathrm{O} \rightarrow 4 \mathrm{CO}+9 \mathrm{H}_{2}$ \\
\hline 23 & Pentane SR & $\mathrm{C}_{5} \mathrm{H}_{12}+5 \mathrm{H}_{2} \mathrm{O} \rightarrow 5 \mathrm{CO}+11 \mathrm{H}_{2}$ \\
\hline \multicolumn{3}{|c|}{ Alkane partial oxidation } \\
\hline 24 & Metane PO & $\mathrm{CH}_{4}+0.5 \mathrm{O}_{2} \rightarrow \mathrm{CO}+2 \mathrm{H}_{2}$ \\
\hline 25 & Etane PO & $\mathrm{C}_{2} \mathrm{H}_{6}+\mathrm{O}_{2} \rightarrow 2 \mathrm{CO}+3 \mathrm{H}_{2}$ \\
\hline 26 & Propane PO & $\mathrm{C}_{3} \mathrm{H}_{8}+1.5 \mathrm{O}_{2} \rightarrow 3 \mathrm{CO}+4 \mathrm{H}_{2}$ \\
\hline 27 & Butane PO & $\mathrm{C}_{4} \mathrm{H}_{10}+2 \mathrm{O}_{2} \rightarrow 4 \mathrm{CO}+5 \mathrm{H}_{2}$ \\
\hline 28 & Pentane PO & $\mathrm{C}_{5} \mathrm{H}_{12}+2.5 \mathrm{O}_{2} \rightarrow 5 \mathrm{CO}+6 \mathrm{H}_{2}$ \\
\hline \multicolumn{3}{|c|}{ Alkene steam reforming } \\
\hline 29 & Ethylene SR & $\mathrm{C}_{2} \mathrm{H}_{4}+2 \mathrm{H}_{2} \mathrm{O} \rightarrow 2 \mathrm{CO}+4 \mathrm{H}_{2}$ \\
\hline 30 & Propylene SR & $\mathrm{C}_{3} \mathrm{H}_{6}+3 \mathrm{H}_{2} \mathrm{O} \rightarrow 3 \mathrm{CO}+6 \mathrm{H}_{2}$ \\
\hline 31 & Butene SR & $\mathrm{C}_{4} \mathrm{H}_{8}+4 \mathrm{H}_{2} \mathrm{O} \rightarrow 4 \mathrm{CO}+8 \mathrm{H}_{2}$ \\
\hline 32 & Pentene SR & $\mathrm{C}_{5} \mathrm{H}_{10}+5 \mathrm{H}_{2} \mathrm{O} \rightarrow 5 \mathrm{CO}+10 \mathrm{H}_{2}$ \\
\hline \multicolumn{3}{|c|}{ Alkene partial oxidation } \\
\hline 33 & Ethylene PO & $\mathrm{C}_{2} \mathrm{H}_{4}+\mathrm{O}_{2} \rightarrow 2 \mathrm{CO}+2 \mathrm{H}_{2}$ \\
\hline 34 & Propylene PO & $\mathrm{C}_{3} \mathrm{H}_{6}+1.5 \mathrm{O}_{2} \rightarrow 3 \mathrm{CO}+3 \mathrm{H}_{2}$ \\
\hline 35 & Butene PO & $\mathrm{C}_{4} \mathrm{H}_{8}+2 \mathrm{O}_{2} \rightarrow 4 \mathrm{CO}+4 \mathrm{H}_{2}$ \\
\hline 36 & Pentene PO & $\mathrm{C}_{5} \mathrm{H}_{10}+2.5 \mathrm{O}_{2} \rightarrow 5 \mathrm{CO}+5 \mathrm{H}_{2}$ \\
\hline
\end{tabular}




\begin{tabular}{lll}
\hline \multicolumn{1}{l}{ No. } & \multicolumn{1}{c}{ Reaction } \\
\hline \multicolumn{2}{l}{ Alkane oxidation } & \\
\hline 37 & Methane oxidation & $\mathrm{CH}_{4}+2 \mathrm{O}_{2} \rightarrow \mathrm{CO}_{2}+2 \mathrm{H}_{2} \mathrm{O}$ \\
38 & Ethane oxidation & $\mathrm{C}_{2} \mathrm{H}_{4}+2 \mathrm{O}_{2} \rightarrow \mathrm{CO}_{2}+2 \mathrm{H}_{2} \mathrm{O}$ \\
39 & Propane oxidation & $\mathrm{C}_{3} \mathrm{H}_{8}+5 \mathrm{O}_{2} \rightarrow 3 \mathrm{CO}_{2}+4 \mathrm{H}_{2} \mathrm{O}$ \\
40 & Butane oxidation & $\mathrm{C}_{4} \mathrm{H}_{10}+6.5 \mathrm{O}_{2} \rightarrow 4 \mathrm{CO}_{2}+5 \mathrm{H}_{2} \mathrm{O}$ \\
41 & Pentane oxidation & $\mathrm{C}_{5} \mathrm{H}_{12}+8 \mathrm{O}_{2} \rightarrow 5 \mathrm{CO}_{2}+6 \mathrm{H}_{2} \mathrm{O}$ \\
\hline \multicolumn{2}{l}{ Decomposition } & \\
\hline 42 & Palmitic acid decomposition(1) & $\mathrm{C}_{16} \mathrm{H}_{32} \mathrm{O}_{2} \rightarrow 2 \mathrm{H}_{2}+7 \mathrm{CH}_{4}+7 \mathrm{C}+2 \mathrm{CO}$ \\
43 & Palmitic acid decomposition(2) & $\mathrm{C}_{16} \mathrm{H}_{32} \mathrm{O}_{2} \rightarrow 2 \mathrm{H}_{2}+7 \mathrm{CH}_{4}+8 \mathrm{C}+\mathrm{CO}_{2}$ \\
\hline
\end{tabular}

\subsection{Estimate Gibbs Free Energy}

Gibbs free energy of each element was calculated from enthalpy, entropy and heat capacity at various temperatures as shown in Eq. (1), using PTC Mathcad Prime 2.0.

$$
\begin{gathered}
\Delta G=\Delta H-T \cdot \Delta S \\
\Delta H=\Delta H_{f}^{o}+\int_{T_{0}}^{T} C_{p_{0}} d T \\
\Delta S=\frac{1}{T_{0}}\left(\Delta H_{f}^{o}-\Delta G_{f}^{o}\right)+\int_{T_{0}}^{T} \frac{C_{p_{0}}}{T} d T \\
C_{p_{0}}=A+B \cdot T+C \cdot T^{2}+D \cdot T^{3}+E \cdot T^{4} \\
\Delta G_{r}=\sum_{i=1}^{N} \gamma_{i} \Delta G_{i} \\
\Delta H_{r}=\sum_{i=1}^{N} \gamma_{i} \Delta H_{i} \\
\Delta S_{r}=\sum_{i=1}^{N} \gamma_{i} \Delta S_{i}
\end{gathered}
$$

( $i=$ component, $\gamma_{i}<0$ for reactant component and $\gamma_{i}>0$ for product component)

where $\Delta G$ is the Gibbs free energy of component in $\mathrm{J} / \mathrm{mol}, \Delta H$ is the enthalpy of component in $\mathrm{J} / \mathrm{mol}$, $\Delta S$ is the entropy of component in $\mathrm{J} / \mathrm{mol} \mathrm{K}, C_{p_{0}}$ is the capacity of component in $\mathrm{J} / \mathrm{g} \mathrm{mol} \mathrm{K}, \Delta G_{r}$, $\Delta H_{r}$ and $\Delta S_{r}$ are the Gibbs free energy in $\mathrm{J} / \mathrm{mol}$, the enthalpy in $\mathrm{J} / \mathrm{mol}$ and the entropy of reaction in $\mathrm{J} / \mathrm{mol} \mathrm{K}$, respectively. A, B, C, D and E are characteristic constants of particular gas taken from Handbook of Thermodynamics Diagram Volume 1-4 [12] at each operating temperature, which were determined by Aspen Plus using RGibb reactor and SRK equation.

Equilibrium constant $(K)$ was calculated by the following equation:

$$
K=e^{\left(\frac{-\Delta G}{R T}\right)}
$$

where $K$ is the chemical reaction equilibrium constant for reaction, $\Delta G$ is the Gibbs free energy in $\mathrm{kJ} / \mathrm{mol}, R$ is the universal gas constant in $\mathrm{J} / \mathrm{mol} \mathrm{K}$ and $T$ is the temperature in $\mathrm{K}$.

\subsection{Computational Prediction}

The yield and duty of each temperature were calculated from Aspen Plus program using RGibb reactor and SRK equation. Molar feed ratios of steam to palmitic acid (S/C) and oxygen to palmitic acid (O/C) are 0.5 to 4 , respectively, with autothermal reaction at ambient pressure and temperatures between $800-1000^{\circ} \mathrm{C}$. Methanation of CO synthesis is a catalytic exothermal process at temperatures of $473-673 \mathrm{~K}\left(200-400^{\circ} \mathrm{C}\right)$ and high pressure does not have any effect on hydrogen and carbon monoxide yields [13-17].

Hydrogen yield was calculated using Eq. (9) 


$$
Y_{i}=\frac{F_{\text {iout }}}{F_{P_{\text {in }}}}
$$

where $Y_{i}$ is the yield of hydrogen, $F_{\text {iout }}$ is the molar flow rate of hydrogen at outlet, $F_{P_{\text {in }}}$ is the molar flow rate of palmitic acid at inlet.

\section{Results and Discussion}

\subsection{Estimate Gibbs Free Energy of Reaction}

PTC Mathcad Prime 2.0 program is used for component enthalpy calculation where $\Delta G_{f}$ and $\Delta H_{f}$ are the standard Gibbs free energy of formation and enthalpy of formation in $\mathrm{J} / \mathrm{mol}$, respectively [18], and temperature in ${ }^{\circ} \mathrm{C}$.

Evaluation of the enthalpy, entropy and Gibbs free energy were carried out using Eq. (5) to Eq. (7), to predict the thermodynamic behavior of the palmitic acid autothermal reforming. Estimated results are shown in Table 2. Equilibrium constants were estimated using Eq. (8). Heat of reactions, difference between the total enthalpy of formation of the products and that of the reactant, were calculated to indicate whether it is endothermic or exothermic reaction [19]. For the endothermic reaction, where the heat of reaction is positive, sufficient energy from surrounding is required to drive the process forward. On the other hand, the reaction is considered to be exothermic if it shows negative heat of reaction. In this case, the internal energy needs to be released to the surrounding during the reaction. In addition, Gibbs free energy of reaction can determine the direction of reaction, for example, negative of Gibbs free energy indicates that the reaction proceeds spontaneously in the forward direction at a particular temperature and pressure while the reaction proceeds spontaneously in the reverse direction for positive of Gibbs free energy [19].

From the result, most of reactions in the autothermal process are exothermic at these three temperatures except the steam reforming of palmitic acid (R-1), overall reaction of palmitic acid (R-3), methane decomposition (R-4), methane steam reforming (R-11), methane dry reforming (R-12), reverse water-gas shift reaction (R-15), coke gasification (R-16), alkane steam reforming (R-19 to 23) and alkene steam reforming (R-29 to 32). From the Gibbs free energy, the boudouard reaction (R-5), hydrogenation of carbon monoxide (1) and (2) (R-7 and R-9), hydrogenation of carbon dioxide (R-8), hydrogenation of coke (R-10), and carbon monoxide reduction (1) and (2) (R-13 to 14) are proceeded in the reverse reaction. Interestingly, water-gas shift reaction (R-6) is spontaneously forward reaction at low temperature $\left(800^{\circ} \mathrm{C}\right)$ while at higher temperature $\left(900\right.$ and $\left.1000^{\circ} \mathrm{C}\right)$, it becomes spontaneously reverse reaction [20].

Once the Gibbs free energy is obtained, the thermodynamic equilibrium constant of the reaction $(K)$ is then calculated using Eq. (8) to estimate the extent of the reaction. When the thermodynamic equilibrium constant is larger than 1.0, it indicates that the mixture at the process outlet contains mostly products, while less than 1.0 of that states the reactants as the main components. However, if the thermodynamic equilibrium constant is equal to 1.0 , it means that the reaction reaches equilibrium with the same amounts of products and reactants.

Table 3 show the spontaneous reaction and $K$ value of three temperatures using Eq. (8). The result shows that reactive reactions are boudouard reaction (R-5), hydrogenation of carbon dioxide (R-8), carbon monoxide reduction (1) (R-7), carbon monoxide reduction (2) (R-9) and hydrogenation of coke (R-10). Water-gas shift reaction (R-6) is active at $800^{\circ} \mathrm{C}$ while reverse water-gas shift reaction (R-15) is active at 900 and $1000^{\circ} \mathrm{C}$. Gibbs free energy of the water-gas shift reaction was increased with increasing temperature and became positive (cannot be occurred by itself) at temperature around $900^{\circ} \mathrm{C}[20]$. 
Table 2. Enthalpy of reaction.

\begin{tabular}{|c|c|c|c|c|c|c|c|}
\hline \multirow[b]{2}{*}{ No. } & \multirow[b]{2}{*}{ Reaction } & \multicolumn{2}{|c|}{ At $800^{\circ} \mathrm{C}$} & \multicolumn{2}{|c|}{ At $900^{\circ} \mathrm{C}$} & \multicolumn{2}{|c|}{ At $1000^{\circ} \mathrm{C}$} \\
\hline & & $\begin{array}{c}\Delta H \\
(\mathrm{~J} / \mathrm{mol})\end{array}$ & $\begin{array}{c}\Delta G \\
(\mathrm{~J} / \mathrm{mol})\end{array}$ & $\begin{array}{c}\Delta H \\
(\mathrm{~J} / \mathrm{mol})\end{array}$ & $\begin{array}{c}\Delta G \\
(\mathrm{~J} / \mathrm{mol})\end{array}$ & $\begin{array}{c}\Delta H \\
(\mathrm{~J} / \mathrm{mol})\end{array}$ & $\begin{array}{c}\Delta G \\
(\mathrm{~J} / \mathrm{mol})\end{array}$ \\
\hline 1 & Steam reforming of palmitic acid & $1.87 \times 10^{6}$ & $-2.31 \times 10^{5}$ & $1.84 \times 10^{6}$ & $-4.41 \times 10^{5}$ & $1.79 \times 10^{6}$ & $-6.50 \times 10^{5}$ \\
\hline 2 & Partial oxidation of palmitic acid & $-5.57 \times 10^{6}$ & $-6.54 \times 10^{6}$ & $-5.62 \times 10^{6}$ & $-6.64 \times 10^{6}$ & $-5.69 \times 10^{6}$ & $-6.74 \times 10^{6}$ \\
\hline 3 & Overall reaction of peroxide & $-2.02 \times 10^{6}$ & $-6.23 \times 10^{6}$ & $2.00 \times 10^{6}$ & $-3.63 \times 10^{5}$ & $-2.01 \times 10^{6}$ & $-7.02 \times 10^{6}$ \\
\hline 4 & Methane decomposition & $8.98 \times 10^{4}$ & $-2.76 \times 10^{4}$ & $9.04 \times 10^{4}$ & $-3.86 \times 10^{4}$ & $8.93 \times 10^{4}$ & $-5.11 \times 10^{4}$ \\
\hline 5 & Boudouard reaction & $-1.70 \times 10^{5}$ & $1.73 \times 10^{4}$ & $-1.69 \times 10^{5}$ & $3.46 \times 10^{4}$ & $-1.70 \times 10^{5}$ & $5.04 \times 10^{4}$ \\
\hline 6 & Water- gas shift reaction & $-3.45 \times 10^{4}$ & $-1.10 \times 10^{3}$ & $-3.35 \times 10^{4}$ & $1.96 \times 10^{3}$ & $-3.25 \times 10^{4}$ & $4.94 \times 10^{3}$ \\
\hline 7 & Hydrogenation of carbon monoxide(1) & $-2.26 \times 10^{5}$ & $4.60 \times 10^{4}$ & $-2.26 \times 10^{5}$ & $7.12 \times 10^{4}$ & $-2.27 \times 10^{5}$ & $9.66 \times 10^{4}$ \\
\hline 8 & Hydrogenation of carbon dioxide & $-1.91 \times 10^{5}$ & $4.71 \times 10^{4}$ & $-1.93 \times 10^{5}$ & $6.92 \times 10^{4}$ & $-1.94 \times 10^{5}$ & $9.16 \times 10^{4}$ \\
\hline 9 & Hydrogenation of carbon monoxide(2) & $-2.60 \times 10^{5}$ & $4.49 \times 10^{4}$ & $-2.60 \times 10^{5}$ & $7.32 \times 10^{4}$ & $-2.59 \times 10^{5}$ & $1.02 \times 10^{5}$ \\
\hline 10 & Hydrogenation of coke & $-8.98 \times 10^{4}$ & $2.76 \times 10^{4}$ & $-9.04 \times 10^{4}$ & $3.86 \times 10^{4}$ & $-8.93 \times 10^{4}$ & $5.11 \times 10^{4}$ \\
\hline 11 & Methane steam reforming & $2.26 \times 10^{5}$ & $-4.60 \times 10^{4}$ & $2.26 \times 10^{5}$ & $-7.12 \times 10^{4}$ & $2.27 \times 10^{5}$ & $-9.66 \times 10^{4}$ \\
\hline 12 & Methane dry reforming & $2.60 \times 10^{5}$ & $-4.49 \times 10^{4}$ & $2.60 \times 10^{5}$ & $-7.32 \times 10^{4}$ & $2.59 \times 10^{5}$ & $-1.02 \times 10^{5}$ \\
\hline 13 & Carbon monoxide reduction(1) & $-1.36 \times 10^{5}$ & $1.84 \times 10^{4}$ & $-1.36 \times 10^{5}$ & $3.26 \times 10^{4}$ & $-1.37 \times 10^{5}$ & $4.55 \times 10^{4}$ \\
\hline 14 & Carbon monoxide reduction(2) & $-2.72 \times 10^{5}$ & $3.67 \times 10^{4}$ & $-2.72 \times 10^{5}$ & $6.53 \times 10^{4}$ & $-2.74 \times 10^{5}$ & $9.09 \times 10^{4}$ \\
\hline 15 & Reverse water-gas shift reaction & $3.45 \times 10^{4}$ & $1.10 \times 10^{3}$ & $3.35 \times 10^{4}$ & $-1.96 \times 10^{3}$ & $3.25 \times 10^{4}$ & $-4.94 \times 10^{3}$ \\
\hline 16 & Coke gasification & $1.36 \times 10^{5}$ & $-1.84 \times 10^{4}$ & $1.36 \times 10^{5}$ & $-3.26 \times 10^{4}$ & $1.37 \times 10^{5}$ & $-4.55 \times 10^{4}$ \\
\hline 17 & Oxidation of methane & $-8.02 \times 10^{5}$ & $-8.01 \times 10^{5}$ & $-8.03 \times 10^{5}$ & $-8.02 \times 10^{5}$ & $-8.03 \times 10^{5}$ & $-8.02 \times 10^{5}$ \\
\hline \multirow[t]{2}{*}{18} & Partial oxidation of carbon monoxide & $-2.83 \times 10^{5}$ & $-1.90 \times 10^{5}$ & $-2.82 \times 10^{5}$ & $-1.81 \times 10^{5}$ & $-2.82 \times 10^{5}$ & $-1.73 \times 10^{5}$ \\
\hline & Alkane steam reforming & & & & & & \\
\hline 19 & Methane SR & $2.26 \times 10^{5}$ & $-4.60 \times 10^{4}$ & $2.26 \times 10^{5}$ & $-7.12 \times 10^{4}$ & $2.27 \times 10^{5}$ & $-9.66 \times 10^{4}$ \\
\hline 20 & Ethane SR & $2.52 \times 10^{5}$ & $-2.89 \times 10^{5}$ & $1.99 \times 10^{5}$ & $-3.94 \times 10^{5}$ & $3.74 \times 10^{5}$ & $-2.68 \times 10^{5}$ \\
\hline 21 & Propane SR & $5.38 \times 10^{5}$ & $-2.71 \times 10^{5}$ & $5.38 \times 10^{5}$ & $-3.46 \times 10^{5}$ & $5.38 \times 10^{5}$ & $-4.21 \times 10^{5}$ \\
\hline 22 & Butane SR & $7.10 \times 10^{5}$ & $-4.23 \times 10^{5}$ & $6.99 \times 10^{5}$ & $-5.40 \times 10^{5}$ & $6.98 \times 10^{5}$ & $-6.45 \times 10^{5}$ \\
\hline \multirow[t]{2}{*}{23} & Pentane SR & $8.60 \times 10^{5}$ & $-5.14 \times 10^{5}$ & $8.59 \times 10^{5}$ & $-6.41 \times 10^{5}$ & $8.58 \times 10^{5}$ & $-7.69 \times 10^{5}$ \\
\hline & Alkane partial os & & & & & & \\
\hline 24 & Methane PO & $-2.25 \times 10^{4}$ & $-2.35 \times 10^{5}$ & $-2.26 \times 10^{4}$ & $-2.54 \times 10^{5}$ & $-2.28 \times 10^{4}$ & $-2.74 \times 10^{5}$ \\
\hline 25 & Ethane PO & $-2.44 \times 10^{5}$ & $-6.66 \times 10^{5}$ & $-2.99 \times 10^{5}$ & $-7.60 \times 10^{5}$ & $-1.24 \times 10^{5}$ & $-6.23 \times 10^{5}$ \\
\hline 26 & Propane PO & $-2.07 \times 10^{5}$ & $-8.36 \times 10^{5}$ & $-2.08 \times 10^{5}$ & $-8.95 \times 10^{5}$ & $-2.10 \times 10^{5}$ & $-9.53 \times 10^{5}$ \\
\hline 27 & Butane PO & $-2.83 \times 10^{5}$ & $-1.18 \times 10^{6}$ & $-2.96 \times 10^{5}$ & $-1.27 \times 10^{6}$ & $-2.99 \times 10^{5}$ & $-1.36 \times 10^{6}$ \\
\hline \multirow[t]{2}{*}{28} & Pentane PO & $-3.82 \times 10^{5}$ & $-1.46 \times 10^{6}$ & $-3.85 \times 10^{5}$ & $-1.56 \times 10^{6}$ & $-3.89 \times 10^{5}$ & $-1.66 \times 10^{6}$ \\
\hline & & & & & & & \\
\hline 29 & Ethylene SR & $2.34 \times 10^{5}$ & $-1.62 \times 10^{5}$ & $2.35 \times 10^{5}$ & $-1.99 \times 10^{5}$ & $2.35 \times 10^{5}$ & $-2.36 \times 10^{5}$ \\
\hline 30 & Propylene SR & $4.08 \times 10^{5}$ & $-2.54 \times 10^{4}$ & $4.09 \times 10^{5}$ & $-6.56 \times 10^{4}$ & $4.09 \times 10^{5}$ & $-1.06 \times 10^{5}$ \\
\hline 31 & Butene SR & $5.69 \times 10^{5}$ & $-1.01 \times 10^{5}$ & $5.69 \times 10^{5}$ & $-1.63 \times 10^{5}$ & $5.69 \times 10^{5}$ & $-2.26 \times 10^{5}$ \\
\hline \multirow[t]{2}{*}{32} & Pentene SR & $7.30 \times 10^{5}$ & $-1.80 \times 10^{5}$ & $7.30 \times 10^{5}$ & $-2.64 \times 10^{5}$ & $7.30 \times 10^{5}$ & $-3.49 \times 10^{5}$ \\
\hline & Alkene partial oxidation & & & & & & \\
\hline 33 & Ethylene PO & $-2.63 \times 10^{5}$ & $-5.39 \times 10^{5}$ & $-2.63 \times 10^{5}$ & $-5.65 \times 10^{5}$ & $-2.64 \times 10^{5}$ & $-5.91 \times 10^{5}$ \\
\hline 34 & Propylene PO & $-3.36 \times 10^{5}$ & $-5.91 \times 10^{5}$ & $-3.38 \times 10^{5}$ & $-6.15 \times 10^{5}$ & $-3.39 \times 10^{5}$ & $-6.39 \times 10^{5}$ \\
\hline 35 & Butene PO & $-4.24 \times 10^{5}$ & $-8.55 \times 10^{5}$ & $-4.26 \times 10^{5}$ & $-8.96 \times 10^{5}$ & $-4.29 \times 10^{5}$ & $-9.36 \times 10^{5}$ \\
\hline \multirow[t]{2}{*}{36} & Pentene PO & $-5.11 \times 10^{5}$ & $-1.12 \times 10^{6}$ & $-5.14 \times 10^{5}$ & $-1.18 \times 10^{6}$ & $-5.17 \times 10^{5}$ & $-1.24 \times 10^{6}$ \\
\hline & Alkane oxidation & & & & & & \\
\hline 37 & Methane oxidation & $-8.02 \times 10^{5}$ & $-8.01 \times 10^{5}$ & $-8.03 \times 10^{5}$ & $-8.02 \times 10^{5}$ & $-8.03 \times 10^{5}$ & $-8.02 \times 10^{5}$ \\
\hline 38 & Ethane oxidation & $-1.55 \times 10^{6}$ & $-1.61 \times 10^{6}$ & $-1.61 \times 10^{6}$ & $-1.67 \times 10^{6}$ & $-1.44 \times 10^{6}$ & $-1.50 \times 10^{6}$ \\
\hline 39 & Propane oxidation & $-2.05 \times 10^{6}$ & $-2.16 \times 10^{6}$ & $-2.05 \times 10^{6}$ & $-2.17 \times 10^{6}$ & $-2.05 \times 10^{6}$ & $-2.18 \times 10^{6}$ \\
\hline 40 & Butane oxidation & $-2.65 \times 10^{6}$ & $-2.88 \times 10^{6}$ & $-2.67 \times 10^{6}$ & $-2.91 \times 10^{6}$ & $-2.67 \times 10^{6}$ & $-2.93 \times 10^{6}$ \\
\hline \multirow[t]{2}{*}{41} & Pentane oxidation & $-3.28 \times 10^{6}$ & $-3.54 \times 10^{6}$ & $-3.29 \times 10^{6}$ & $-3.56 \times 10^{6}$ & $-3.29 \times 10^{6}$ & $-3.58 \times 10^{6}$ \\
\hline & Decomposition & & & & & & \\
\hline 42 & Palmitic acid decomposition(1) & $-6.58 \times 10^{5}$ & $-3.15 \times 10^{5}$ & $-6.94 \times 10^{5}$ & $-2.81 \times 10^{5}$ & $-7.53 \times 10^{5}$ & $-2.55 \times 10^{5}$ \\
\hline 43 & Palmitic acid decomposition(2) & $-8.28 \times 10^{5}$ & $-2.98 \times 10^{5}$ & $-8.63 \times 10^{5}$ & $-2.47 \times 10^{5}$ & $-9.22 \times 10^{5}$ & $-2.05 \times 10^{5}$ \\
\hline
\end{tabular}


Table 3. Thermodynamic equilibrium constant for autothermal reforming reactions using palmitic acid.

\begin{tabular}{|c|c|c|c|c|}
\hline \multirow{2}{*}{ No. } & \multirow{2}{*}{ Reaction } & \multicolumn{3}{|c|}{ Equilibrium constant $(K)$} \\
\hline & & At $800^{\circ} \mathrm{C}$ & At $900^{\circ} \mathrm{C}$ & At $1000^{\circ} \mathrm{C}$ \\
\hline 1 & Steam reforming of palmitic acid & 1.09 & 1.11 & 1.14 \\
\hline 2 & Partial oxidation of palmitic acid & 2.05 & 1.95 & 1.96 \\
\hline 3 & Overall reaction of palmitic acid & 1.50 & 1.47 & 1.49 \\
\hline 4 & Methane decomposition & 1.00 & 1.00 & 1.01 \\
\hline 5 & Boudouard reaction & * & $*$ & * \\
\hline 6 & Water- gas shift reaction & 1.00 & * & * \\
\hline 7 & Hydrogenation of carbon monoxide(1) & $*$ & $*$ & $*$ \\
\hline 8 & Hydrogenation of carbon dioxide & * & * & * \\
\hline 9 & Hydrogenation of carbon monoxide(2) & * & * & * \\
\hline 10 & Hydrogenation of coke & * & * & * \\
\hline 11 & Methane steam reforming & 1.01 & 1.01 & 1.01 \\
\hline 12 & Methane dry reforming & 1.01 & 1.01 & 1.01 \\
\hline 13 & Carbon monoxide reduction(1) & $*$ & $*$ & $*$ \\
\hline 14 & Carbon monoxide reduction(2) & $*$ & $*$ & $*$ \\
\hline 15 & Reverse water-gas shift reaction & $*$ & 1.00 & 1.00 \\
\hline 16 & Coke gasification & 1.00 & 1.00 & 1.00 \\
\hline 17 & Oxidation of methane & 1.09 & 1.09 & 1.09 \\
\hline 18 & Partial oxidation of carbon monoxide & 1.02 & 1.02 & 1.02 \\
\hline \multicolumn{5}{|c|}{ Alkane steam reforming } \\
\hline 19 & Methane SR & 1.01 & 1.01 & 1.01 \\
\hline 20 & Ethane SR & 1.03 & 1.04 & 1.03 \\
\hline 21 & Propane SR & 1.03 & 1.04 & 1.04 \\
\hline 22 & Butane SR & 1.05 & 1.06 & 1.07 \\
\hline 23 & Pentane SR & 1.06 & 1.07 & 1.08 \\
\hline \multicolumn{5}{|c|}{ Alkane partial oxidation } \\
\hline 24 & Metane PO & 1.03 & 1.03 & 1.03 \\
\hline 25 & Etane PO & 1.08 & 1.08 & 1.07 \\
\hline 26 & Propane PO & 1.10 & 1.10 & 1.10 \\
\hline 27 & Butane PO & 1.14 & 1.14 & 1.15 \\
\hline 28 & Pentane PO & 1.18 & 1.17 & 1.19 \\
\hline \multicolumn{5}{|c|}{ Alkene steam reforming } \\
\hline 29 & Ethylene SR & 1.03 & 1.02 & 1.02 \\
\hline 30 & Propylene SR & 1.08 & 1.01 & 1.01 \\
\hline 31 & Butene SR & 1.01 & 1.02 & 1.02 \\
\hline 32 & Pentene SR & 1.02 & 1.03 & 1.04 \\
\hline \multicolumn{5}{|c|}{ Alkene partial oxidation } \\
\hline 33 & Ethylene PO & 1.06 & 1.06 & 1.06 \\
\hline 34 & Propylene PO & 1.07 & 1.07 & 1.07 \\
\hline 35 & Butene PO & 1.01 & 1.10 & 1.10 \\
\hline 36 & Pentene PO & 1.13 & 1.13 & 1.14 \\
\hline \multicolumn{5}{|c|}{ Alkane oxidation } \\
\hline 37 & Methane oxidation & 1.09 & 1.09 & 1.09 \\
\hline 38 & Ethane oxidation & 1.20 & 1.21 & 1.18 \\
\hline 39 & Propane oxidation & 1.27 & 1.28 & 1.28 \\
\hline 40 & Butane oxidation & 1.38 & 1.39 & 1.39 \\
\hline 41 & Pentane oxidation & 1.49 & 1.49 & 1.49 \\
\hline \multicolumn{5}{|c|}{ Decomposition } \\
\hline 42 & Palmitic acid decomposition(1) & 1.04 & 1.03 & 1.03 \\
\hline 43 & Palmitic acid decomposition(2) & 1.03 & 1.03 & 1.02 \\
\hline
\end{tabular}




\subsection{Heat Duty Calculation}

The heat duty and yield of hydrogen from steam reforming, partial oxidation reforming and autothermal reforming of palmitic acid using oxygen were determined at three different temperatures, 800, 900 and $1000^{\circ} \mathrm{C}$, under atmospheric pressure using Aspen Plus program where the reactions at each temperature are defined by the minimum Gibbs free energy method. Figure 1 shows heat duties (watts) of autothermal reforming of palmitic acid where the yield of hydrogen is illustrated in Fig. 2.

For all range of S/C ratio (varied from 0.5 to 4), the heat duties of palmitic acid steam reforming decreases with increasing temperature. When the operating temperature was constant, the heat duties show a increasing trend when the ratio $\mathrm{S} / \mathrm{C}$ is increased because the higher $\mathrm{S} / \mathrm{C}$ promotes the endothermic reactions such as steam reforming of palmitic acid (R-1), overall reaction of palmitic acid (R-3), methane decomposition (R-4), methane steam reforming (R-11), methane dry reforming (R-12), coke gasification (R16), alkane steam reforming (R-19 to 23) and alkene steam reforming (R-29 to 32); and prohibit the exothermic reactions such as reaction partial oxidation of palmitic acid (R-2), water-gas shift reaction (R-6), oxidation of methane (R-17), partial oxidation of carbon monoxide (R-18), alkane partial oxidation (R-24 to 28), Alkene partial oxidation (R-33 to 36), alkane oxidation (R-37 to 41) and decomposition of palmitic acid (R-42 and 43).

From the results, higher S/C ratio and lower temperature are favored. The optimum condition was at $\mathrm{S} / \mathrm{C}$ ratio of 3 at $800^{\circ} \mathrm{C}$. Figure 1 shows that the net heat duties of autothermal reforming of palmitic acid. From the graph, the thermoneutral operation conditions can be obtained for S/C of 0.5 and $\mathrm{O} / \mathrm{C}$ of 4 at $800^{\circ} \mathrm{C}$ (408.86 watt with lowest hydrogen yield of 14.94 mole/mole Palmitic $_{\text {) }}$.

The highest endothermic of autothermal reforming using palmitic acid at ratio for S/C of 4 and $\mathrm{O} / \mathrm{C}$ of 0.5 at $1000^{\circ} \mathrm{C}(5,856.78$ watt $)$ can produce a maximum hydrogen yield of 17.88 mole/molepalmitic as shown in Figs. 1 and 2.

The thermodynamic analysis predicted that, during palmitic acid autothermal reforming, hydrogen can be produced from steam reforming of palmitic acid (R-1) in Table 1, overall reaction of palmitic acid (R-3), methane decomposition (R-4), water-gas shift reaction (R-6), methane steam reforming (R-11), methane dry reforming (R-12), coke gasification (R-16), alkane steam reforming (R-19 to 23), alkane partial oxidation (R24 to 28 ), alkene steam reforming (R-29 to 32), alkene partial oxidation (R-33 to 36), alkane oxidation (R37 to 41) and decomposition of palmitic acid (R-42 and 43), whereas hydrogen can be utilized in the reverse water-gas shift reaction (R-15). Figure 2 shows the hydrogen yield as a function of S/C ratio and O/C ratio at different temperatures.

In the palmitic acid autothermal process, carbon monoxide can be produced by steam reforming of palmitic acid (R-1), partial oxidation of palmitic acid (R-2), overall reaction of palmitic acid (R-3), methane steam reforming (R-11), methane dry reforming (R-12), reverse water-gas shift reaction (R-15), coke gasification (R-16), alkane steam reforming (R-19 to 23), alkane partial oxidation (R-24 to 28) and alkene steam reforming (R-29 to 32), alkene partial oxidation (R-33 to 36) and decomposition of palmitic acid (R42 and 43), while it can be consumed in the water-gas shift reaction (R-6) and partial oxidation of carbon (R-18). The carbon monoxide yield as a function of S/C ratio and $\mathrm{O} / \mathrm{C}$ ratio at different temperatures (Fig. 3) can be observed that the yield is increased with increasing temperature as well as increasing both $\mathrm{S} / \mathrm{C}$ and $\mathrm{O} / \mathrm{C}$ ratios. The maximum yield of $7.75 \mathrm{~mole} / \mathrm{molepalmitic}^{\circ}$ and minimum yield of $2.43 \mathrm{~mole} / \mathrm{molep}_{\text {palmitic }}$ can be obtained at $\mathrm{S} / \mathrm{C}$ of 4 and $\mathrm{O} / \mathrm{C}$ of 4 at $1000^{\circ} \mathrm{C}$ and at $\mathrm{S} / \mathrm{C}$ of 0.5 and $\mathrm{O} / \mathrm{C}$ of 0.5 at $800^{\circ} \mathrm{C}$, respectively. 


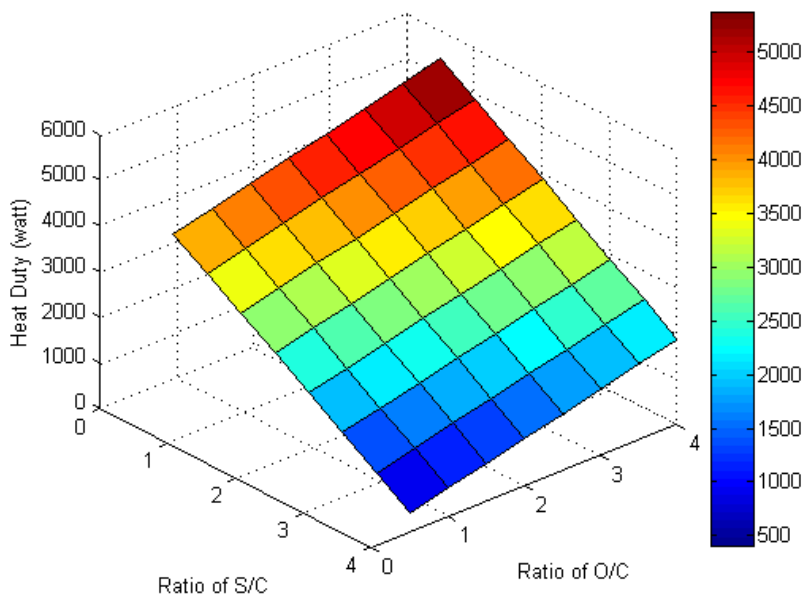

(a)

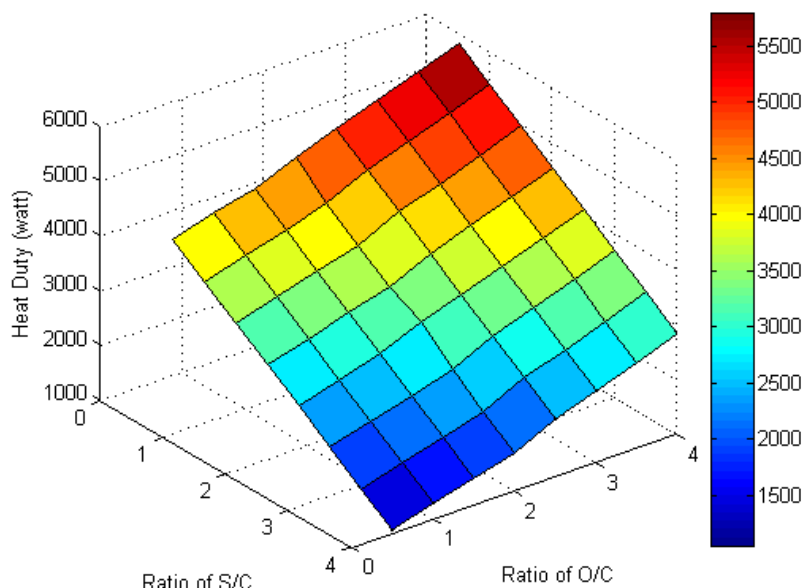

(b)

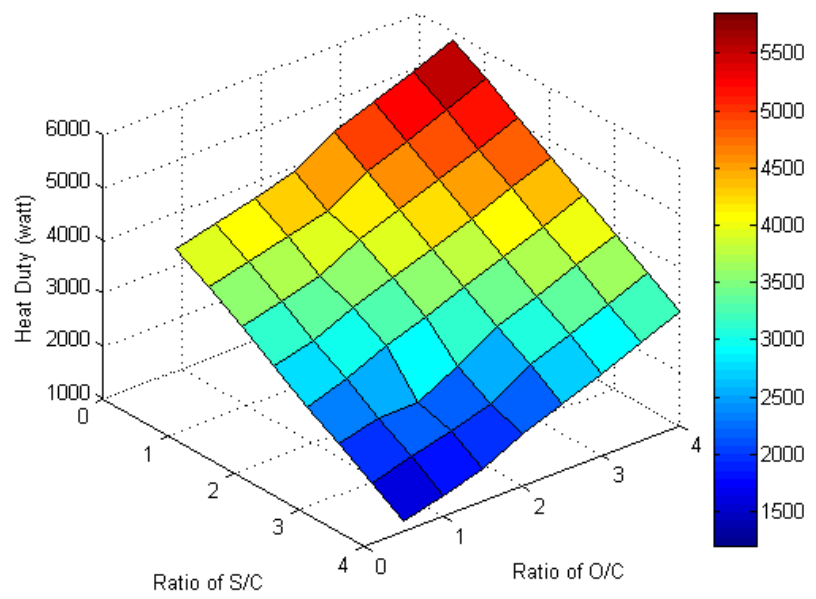

(c)

Fig. 1. Heat duty of autothermal reaction as a function of $\mathrm{S} / \mathrm{C}$ ratio and $\mathrm{O} / \mathrm{C}$ ratio at 1 atm and temperature: (a) $800^{\circ} \mathrm{C}$, (b) $900^{\circ} \mathrm{C}$ and (c) $1000^{\circ} \mathrm{C}$. 


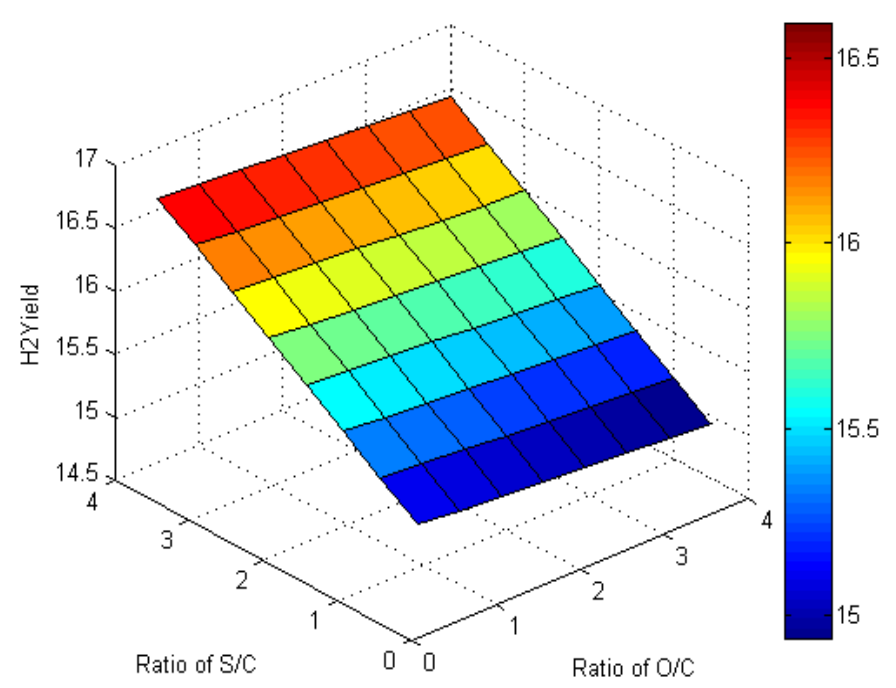

(a)

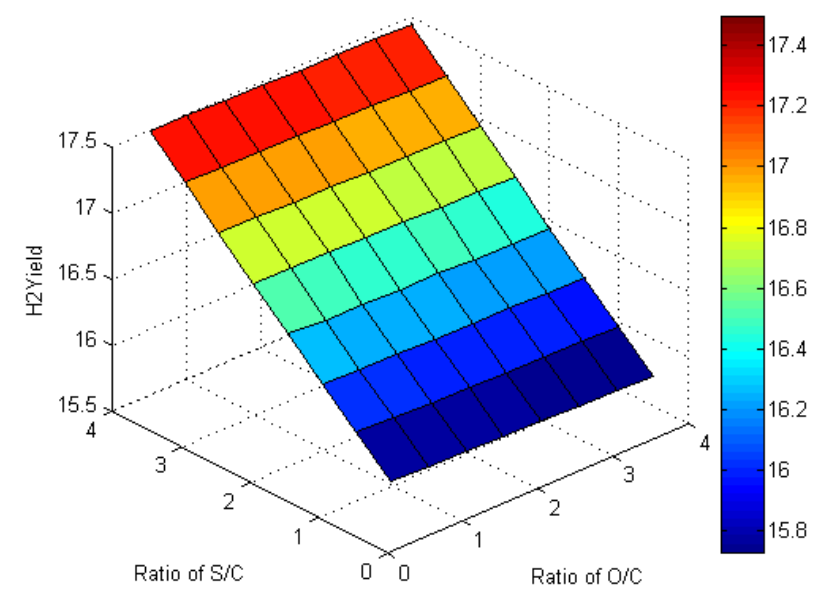

(b)

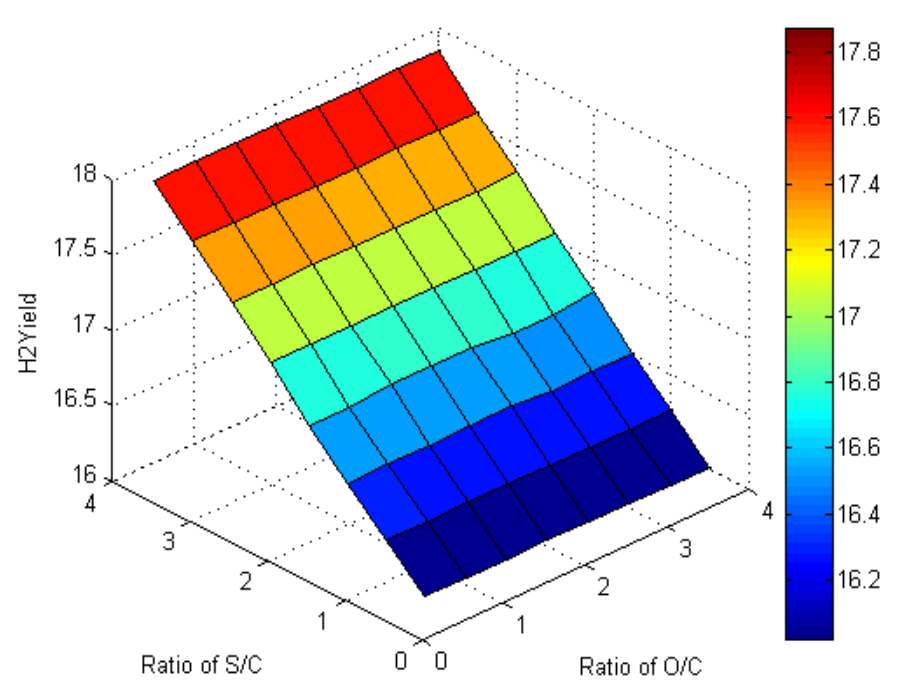

(c)

Fig. 2. Hydrogen yield as a function of $\mathrm{S} / \mathrm{C}$ ratio and $\mathrm{O} / \mathrm{C}$ ratio at $1 \mathrm{~atm}$ and temperature: (a) $800^{\circ} \mathrm{C}$, (b) $900^{\circ} \mathrm{C}$ and (c) $1000^{\circ} \mathrm{C}$. 


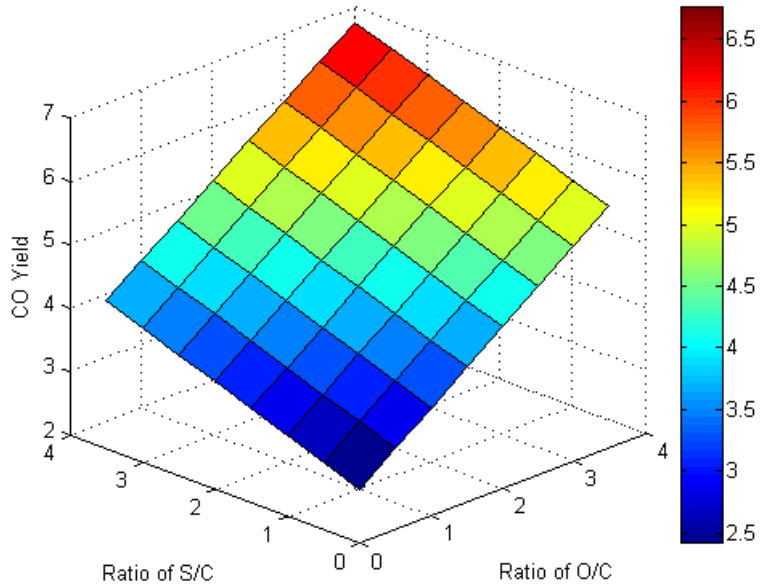

(a)

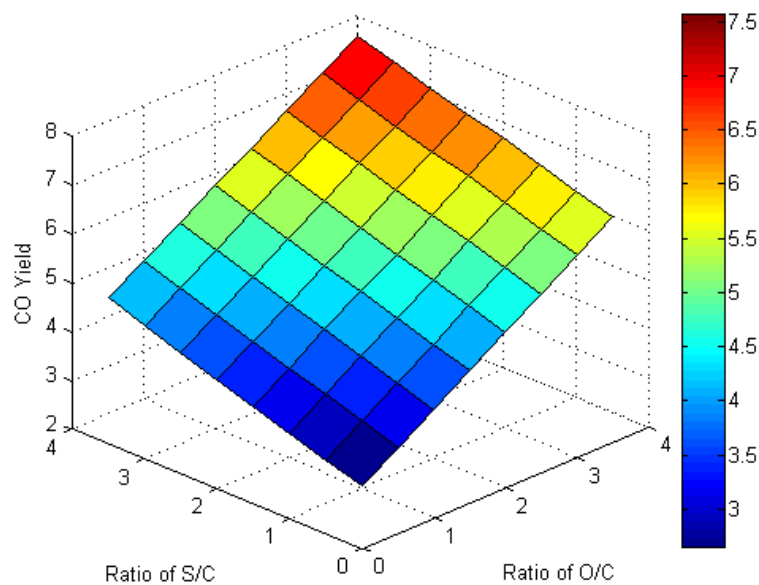

(b)

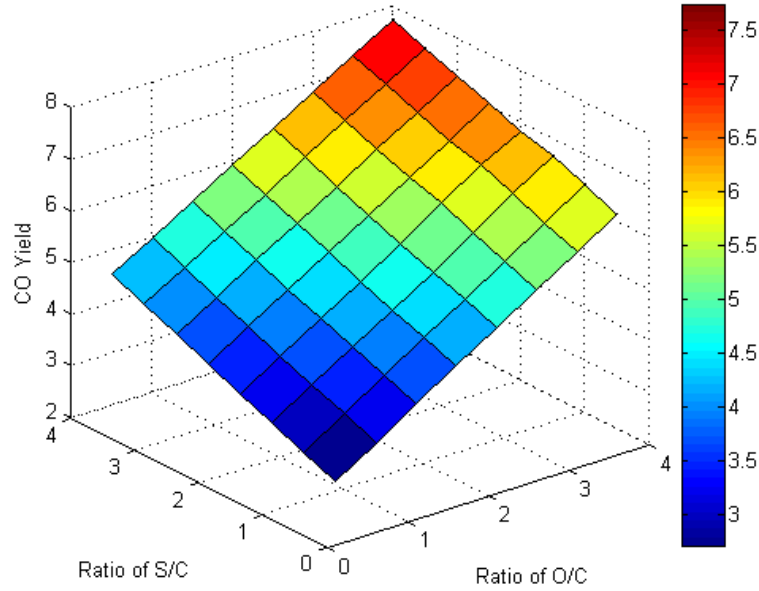

(c)

Fig. 3. Carbon monoxide yield as a function of $\mathrm{S} / \mathrm{C}$ ratio and $\mathrm{O} / \mathrm{C}$ ratio at $1 \mathrm{~atm}$ and temperature: (a) $800^{\circ} \mathrm{C}$, (b) $900^{\circ} \mathrm{C}$ and (c) $1000^{\circ} \mathrm{C}$. 


\section{Conclusions}

Source of hydrogen production can be fossil fuel, higher hydrocarbon and also biomass. Since palmitic acid has high carbon atom, it is suitable to be the reactant for hydrogen production. In this study, the thermodynamic analysis was performed for all possible reactions at each temperature using minimization of the total Gibbs free energy. The net heat duties of steam reforming reaction and partial oxidation reaction decrease when increasing of water ratio and oxygen ratio, respectively. The selected operating temperature strongly influenced the hydrogen yields as well as the heat duty of reaction. Hydrogen production by steam reforming of hydrocarbons, partial oxidation of hydrocarbons and water-gas shift reaction are thermodynamically favored at high temperatures, resulting in higher yields of hydrogen at higher operating temperatures. At low temperatures, hydrogen yield was relatively low due to the formation of alkane by reaction of hydrogen and carbon monoxide. The highest endothermic at ratio for $\mathrm{S} / \mathrm{C}$ of 4 and $\mathrm{O} / \mathrm{C}$ of 0.5 with highest yield of hydrogen was found to be 17.88 mole $/$ mole $_{\text {Palmitic }}$ at $1000^{\circ} \mathrm{C}$.

\section{Acknowledgement}

Grants from Thailand Research Fund of King's Mongkut's University of Technology North Bangkok (MRG5680007) and from King's Mongkut's University of Technology North Bangkok (KMUTNB-GOV58-54, KMUTNB-GOV-57-42) are acknowledged for the support.

\section{References}

[1] J. Larminie and A. Dicks, Fuel Cell Systems Explained, 2nd ed. John Wiley and Sons, 2000.

[2] U. W. Hartley, "Hydrogen-Production and Application," The Journal of Industrial Technology, vol. 9, no. 2, pp. 117-126, 2013.

[3] A. Petchmala, N. Laosiripojana, B. Jongsomjit, M. Goto, J. Panpranot, O. Mekasuwandumrong, and A. Shotipruk, "Transesterification of palm oil and esterification of palm fatty acid in near- and supercritical methanol with $\mathrm{SO}_{4}-\mathrm{ZrO}_{2}$ catalysts," Fuel, vol. 89, pp. 2387-2392, 2010.

[4] P. Mongkolbovornkij, V. Champreda, W. Sutthisripok, and N. Laosiripojana, "Esterification of industrial-grade palm fatty acid distillate over modified $\mathrm{ZrO}_{2}$ (with $\mathrm{WO}^{3-}, \mathrm{SO}^{4}$ and $\mathrm{TiO}^{2-}$ ): Effects of co-solvent adding and water removal," Fuel Processing Technology, vol. 91, pp. 1510-1516, 2010.

[5] A. Shotipruk, S. Assabumrungrat, P. Pavasanta, and N. Laosiripojana, "Reactivity of $\mathrm{CeO}_{2}$ and $\mathrm{Ce}-$ $\mathrm{ZrO}_{2}$ toward steam reforming of palm fatty acid distilled (PFAD) with co-fed oxygenand hydrogen," Chem Eng Sci., vol. 64, pp. 459-466, 2009.

[6] N. Laosiripojana, W. Kiatkittipong, S. Charojrochkul, and S. Assabumrungrat, "Effects of support and co-fed elements on steam reforming of palm fatty acid distillate (PFAD) over Rh-based catalysts," Appl Cat A: General., vol. 383, pp. 50-57, 2010.

[7] N. Laosiripojana, W. Kiatkittipong, and S. Assabumrungrat, "Partial oxidation of palm fatty acids over Ce-ZrO2: Roles of catalyst surface area, lattice oxygen capacity and mobility," AIChE J., vol. 57, pp. 2861-2869, 2011.

[8] E. S. Jung, T. Kim, J. Jin, and S. Kwon, "Autothermal reformer using hydrogen peroxide for micro full cells," in Proc. PowerMEMS 2009, Washington, DC, USA, December 1-4, 2009.

[9] A. N. Gaurav, "Hydrogen rich gas production by the autothermal reforming of biodiesel (FAME) for utilization in the solid-oxide fuel cells: A thermodynamic analysis," Int J of Hydrogen Energy., vol. 35, pp. 8894-8895, 2010.

[10] S. Freni, G. Calogero, and S. Cavallaro, "Hydrogen production from methane through catalytic partial oxidation reactions," J. of Power Sources., vol. 87, pp. 28-38, 2000.

[11] S. H. Chan and H. M. Wang, "Thermodynamic analysis of natural-gas fuel processing for fuel cell applications," Int J of Hydrogen Energy., vol. 25, pp. 441-449, 2000.

[12] C. L. Yaws, Handbook of Thermodynamics Diagram. Texas: Gulf Publishing Company, 1996.

[13] W. H. Chen, M. R. Lin, J. J. Lu, Y. Chao, and T. S. Leu, "Thermodynamic analysis of hydrogen production from methane via autothermal reforming and partial oxidation," Int J of Hydrogen Energy., vol. 35, pp. 787-797, 2010. 
[14] J. C. Amphlett, M. J. Evans, R. A. Jones, R. F. Mann, and R. D. Weir, "Hydrogen production by the catalytic steam reforming of methanol part 1: The thermodynamics," The Canadian J of Chem Eng., vol. 59, pp. 7210-7217, 1981.

[15] K. Ledjeff-Hey, V. Formanski, Th. Kalk, and J. Roes, "Compact hydrogen production systems for solid polymer fuel cells," J of Power Sources., vol. 71, pp. 199-207, 1998.

[16] W. Wenju and Y. Q. Wang, "Thermodynamic analysis of steam reforming of ethanol for hydrogen generation," Int J of Energy Research., vol. 32, pp. 1432-1443, 2008.

[17] G. A. Nahar and S. Y. Madhani., "Thermodynamic of hydrogen production by the steam reforming of butanol: analysis of inorganic gases and light hydrocarbons," Int J of Hydrogen Energy., vol. 35, pp. 98109, 2010.

[18] W. M. Haynes, D. R. Lide, and T. J. Bruno, Handbook of Chemistry and Physics, 93rd ed. New York: CRC Press, 2012.

[19] R. H. Perry and D. W. Green, Perry's Chemical Handbook, 8th ed. New York: McGraw-Hill, 2008.

[20] E. Demirel and N. Azcan, "Thermodynamic modeling of water-gas shift reaction in supercritical water," in Proceedings of the World Congress on Engineering and Computer Science 2012, San Francisco, USA, October 24-26, 2012. 
